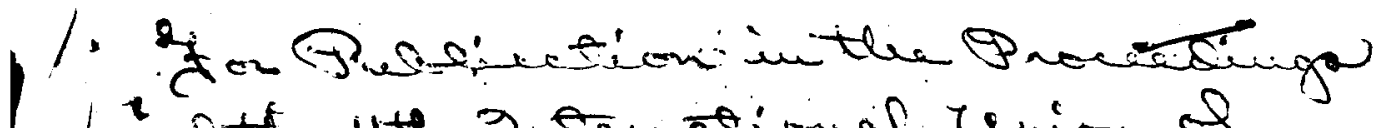

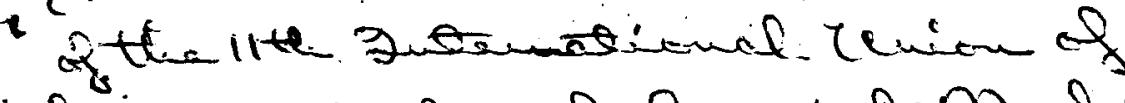

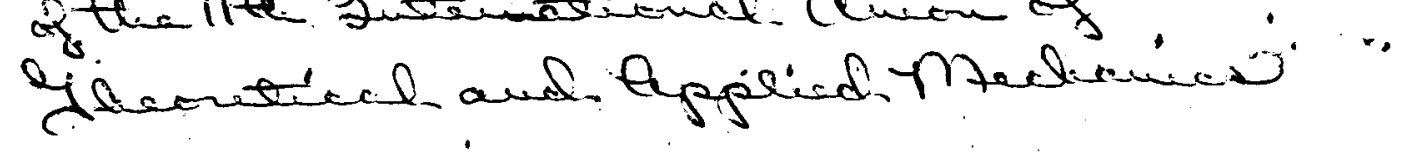

THEORETICAT AND EXPERDMENTAL ASPECTS OF THE SHOCK STRUCTURE PROBLLM"

by

Hans W. Liepmann ${ }^{2}$, Roddam Narasimba ${ }^{2}$ and Moustafa Cbahine ${ }^{3}$

1

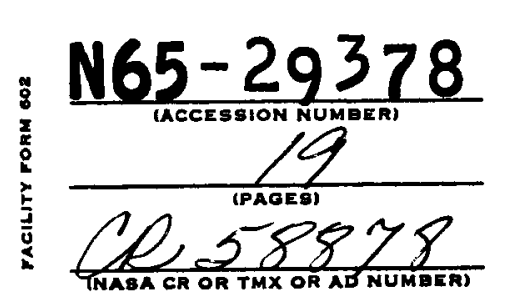

8

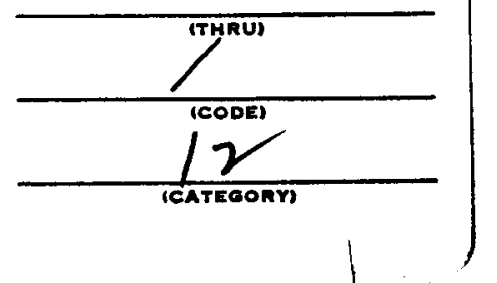

9

GPO PRICE

$\$$

CFSTI PRICE(S) \$

Hard copy $(\mathrm{HC})$

Microfiche (MF)

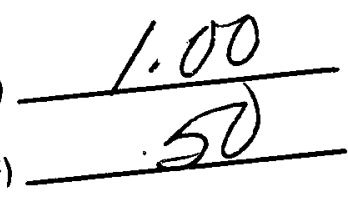

ff 653 July 65

*

This paper presents the results of one phase of research carrled out under Contract No. NAST-200 and Research Grant NsG-40-60 sponsored by the National Aeronautics and Space Administration.

1) California Institute of Technology, Pasadena, California.

2) Indian Institute of ScIence, Bangelore, India.

女3) Jet Propulsion Laboratory, Calffornia Institute of Technology, Pasadena, Calffornia. 
THEORETICAL AND EXPERIMENTAL ASPECTS OF THE SHOCK STRUCTURE PROBLEM

by

Hans W. Liepmann*, Roddam Narasimha** and Moustafa Chahine***

\section{INTRODUCTION AND SUMMARY $:$}

There does not yet exist a general, reliable theory of the

- flow of rarefied gases, bridging the gap between simple free molecular flow methods and the Navier-stokes theory. It is clear today that an essential' improvement of the range of applicability of the Navier-stokes equations cannot be expected from the higher approximations in the Chapman-Enskog procedure but that different methods of finding approximate solutions of the Boltzmann equationf are needed. The shock wave structure problem is one of the best test cases for such attempts because it is realistic amenable. to experimentation, and simple enough to expect that in the future an exact solution of the Boltzmann equation can be obtained.

The present paper discusses essentially recent results obtained by us from an exact numerical solution of the BhathagarGross-Krook model. Short discussions of the Navier-stokes and Mott-Smith theories as well as of recent experimental work are included. The main conclusions reached are the following:

i.) The B-G-K model is capable of describing the essential features of the shock layer and leads to reasonably accurate numerical values for the measurable variables.

ii.) A comparison of the Chapman-Enskog approximation applied to the model and the exact solution show's that useful convergence

\footnotetext{
* California Institute of Technology

** Indian Institute of Science, Bangalore, India

*** Jet Propulsion Laboratory (C.I.T.)
} 
of the C-E series requires stress to pressure ratios, $\tau / p$, less than 0.2, corresponding to shock Mach numbers of less than about 2. iii.) The distribution function within the layer is bimodal, exhibits the gradual change from the molecular beam-like behavior ahead to a Maxwellian distribution behind the shock. The effect of the fast molecules is noticeable even many mean free paths behind the shock. iv.) The flow is nearly. locally adiabatic. That is, the total enthalpy is constant to within/a few per cent.

\section{LOCALLY ADIABATIC FLOW}

The general equations of motion for the flow within a shock wave (Fig. I) can be reduced to a single one, the momentum equation, if one assumes the flow to be "locally adiabatic", i.e., that the heat flux q balances everywhere the work done by the stress " $\mathrm{c}$ i. For "locally adiabatic flow" the total enthalpy $\mathrm{H}=\mathrm{h}+\frac{1}{2} \mathrm{u}^{2}$. is

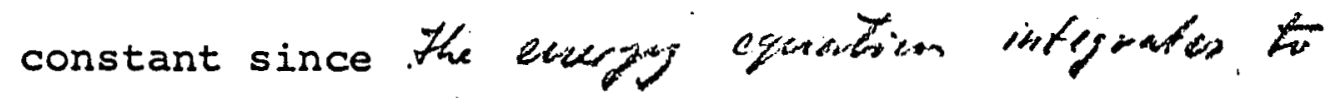

$$
\mathrm{H}-\mathrm{H}_{1}=\tau \mathrm{u}-\dot{\mathrm{q}}
$$

Within the Navier-stokes theory one can easily give an upper bound: for the maximum total enthalpy variation in terms of the Prandtl number Pr:

$$
\left(\frac{\mathrm{H}-\mathrm{H}_{1}}{\mathrm{H}_{1}}\right)_{\max }^{<} \frac{P_{-}-3 / 41}{\gamma(\gamma+1) P_{r}}
$$

In kinetic theory locally adiabatic flow requires a not very stringent relation between the first three moments of the distribution function. Computations for the B-G-K model, shown in Fig. 2 together with the N-S results, demonstrate the comparatively small deviations of $\mathrm{H}$ from $\mathrm{H}_{1}$. The B-G-K model is usally thought 
to imply a Prandtl number of unity; this is correct an the $N-S$ limit, imglief ach

For locally adiabatic flow rife a masplew $m$,

$$
\tau u=\frac{y+1}{2 \gamma} \quad\left(u-u_{1}\right)\left(u-u_{2}\right)=q
$$

and the characteristic parameter $\tau / \mathrm{p}$, can be given explicitly (Fig. 3 ). A. The Navier-stockes theory

The Navier-stokes equations are obtained from the ChapmanEnskog (C-E) approximation if terms proportional to $\frac{\tau}{p}$ are kept. Rapid convergence of the $C-E$ series is thus a sufficient condition for the validity of the $N-S$ equation. The terms of the C-E. series dewever how the form $\frac{\tau}{p} c^{2}$ and $\frac{\tau}{p} M c^{3}$ and hence there exists always regions in velocity space where the series diverges. Indeed for any Mach number there exist, at large $c$, regions for which the C-E distribution function is negative. However this impossible region in the distribution function does not necessarily contribute appreciably to the moments like $n, u$, and $T$. A comparison of the C-E series applied to the B.G.K model and the exact solution indicates a limit of $\frac{\tau}{p} \div 0.2$ for reasonable convergence of the series. A glance at Fig. 3 then shows that one can expect the N-S solution to apply to the complete shock profile for shocks of $\mathrm{M}_{1}<2.0$ approximately, however for 'strong shock waves the $\mathrm{N}-\mathrm{S}$ theory should be applicable only to a relatively small portion

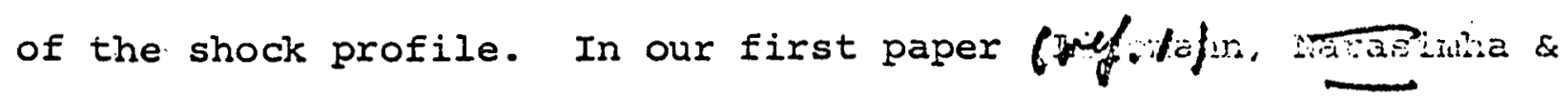
Chanine' I02) we expected the N-s theory to hold for the whole subsonic portion of the shock profile, i.e.. up to $\frac{\tau}{p}$ values of 
0.6 or so. The more precise recent computations reduce the expected applicability of the $\mathrm{N}-\mathrm{S}$ equations and lead to maximum density slope thickness for strong shocks approximately $25 \%$ larger than the corresponding $N-S$ value, not only $10 \%$ as believed in our first paper.

The precise shape of the distribution function is not given correctly by the C-E expansion, for any Mach number. Besides the occurrence of negative regions, the C-E distribution function, due to the non-uniform convergence of the methodifiails to account properly for the fast molecules with long free paths. This leads to an asymptotic approach of, e.g., $u(x)$ to its equilibrium value $u_{1}$ which, Liubarsky (folf $)$ has first shown, is offthe form $u_{n} \sim \exp -|x|^{2 / 3}$ rather than $u_{N S} \sim \exp (-x)$. Since $u_{2} \div u_{N S} \ll u_{N S}$ at least for weak shocks, this behavior is irrelevant for comparison with experiments but of considerable conceptual importance.

B. The Method of Mott-Smith

Mott-Smith was the first to recognize the bimodal character of the distribution function within a shock wave. He proposed an approximate solution of the Boltzmann equation by assuming $f$ to be of the form.

$$
f=[I-\nu(x)] \quad F_{1}+\nu(x) F_{2}
$$

$\nu(x)$ to be determined by a Galerkin-type technique. Mott-Smith' method was certainly an important step in the right direction; however as he himself clearly recognized, the method is limited by 
the arbitrariness in the weighting function used in evaluating $\nu(x)$. Subsequently more elaborate work by Rosen (x/f ), Gustafson ( $r(j)$ and Muckenfuss $(y / f 6)$ doesnot eliminate the arbitrariness in evaluating $\mu(x)$. Sakurai's(rg/5) attempt to prove that the $\mathrm{N}-\mathrm{S}$ method leads to an asymptotically valid solution of the Boltzmann equation for infinite

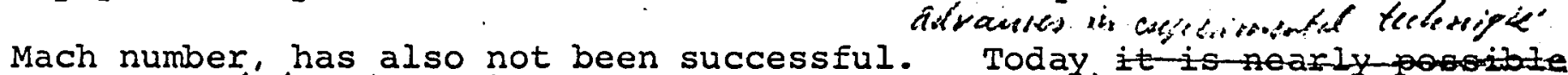

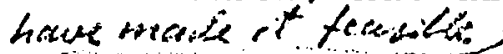
cto chose $\mathcal{\nu}(\mathrm{x})$ for a best fit with experiments. However this would be sensible only if the Mott-Smith approximation could then be developed into a general method in rarefied gas flows. Such an extension does not seem possible.

C. The Bhatnagar-Gross-Krook theory

The model equation we consider is the one proposed by Bhatnagar, Gross \& Krook $(y / g)$. For one-dimensional flow it can be written

$$
v_{x} \frac{\partial f}{\partial x}=A n(F-f)
$$

Equation ( 5 ) is nonlinear in spite of its appearance, as the parameters in the local Maxwellian $F$ areiall moments of the unknown $f$. We have computed an exact numerical solution of (5) satisfying the correct boundary conditions for a shock wave, for, a gas with a realistic viscosity-temperature relation. For the computation it is preferable to convert (5) formally into the integral equation

$$
f\left(x, v_{y^{\prime}} v_{z^{\prime}} v_{x} \gtrless 0\right)=\int_{ \pm \infty}^{x_{j}} \frac{A \cap F}{v_{x}} \cdot \exp \left[-\int_{x^{\prime}}^{x_{x}} \frac{A n d x^{\prime \prime}}{v_{x}}\right] d x^{\prime}
$$

This is now solved by an iterative method whose principles are as follows: A first guess is made at the parameters $n, u$ and $T$ 
(as functions of $\mathrm{x}$ ) appearing in $\mathrm{F}$ on the right hand side; integration with respect to $x$ gives $f$, and further integration with respect to $v$ gives new values for $\rho, u$ and $T$. These are now used to generate the next approximation to $F$, and so on, till there is no sensible variation in the final results. In our computations we used the Navier-stokes solution for shock structure to provide the first guess. Details of the actual computations, the convergence of the scheme and the results obtained were presented ithorifog aric iarasima (1.955).

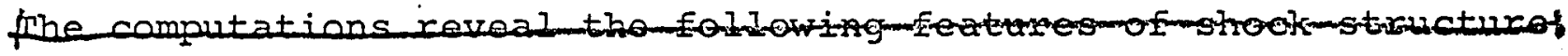
For weak shocks (say $M<2.0$ ), the exact solution hardly differs from the Navier-stokes which is thus an adequate approximation. For stronger shocks the profiles deviate more and more from the NavierStokes, especially on the cold side, where a long tail develops rapidly. On the hot side the iteration converges relatively slowly, and the departure from Navier-stokes is less marked. The thickness of the shock,based on the maximum density slope, is about $25 \%$ larger than the Navier-Stokes value at a Mach number of 10 (and a viscosity law $\mu \sim T^{\omega}$ with $\left.\omega=.816\right)$. The asymmetry in the temperature profile - is very much more pronounced than in the density profile.

We can give a heuristic explanation of these results by considering the form of $f$ as given by' $(6)$. Crucial in determining this is the magnitude of what may be called the "sampling aistance", $\ell=v_{x} / A n$, appearing in the exponential: As $v_{x}$ takes all possible values, so does $\ell$, but all 
velocities $v_{x}$ do not contribute equally to the moments, in which we are' primarily interested.

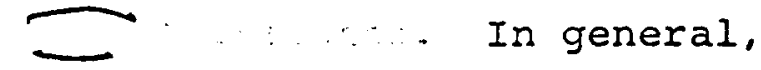
the velocities $v_{x}$ which contriute most to the first few , moments may be expected to be of order $u$. On the hot side of the shock, the Mach number is aiways $O(1)$, or $\dot{u}=O(\bar{c})$. Hence the characteristic sampling distance is $\ell_{2} \sim \bar{c}_{2} / A_{2} n_{2}$, which is proportional to the mean free path $\Lambda_{2}$. Dromexat The gradients within the shock are relatively small. on the hot side over a mean free path so if $F$ varies slowly over the aistance $\ell_{2}$, we may write, using an obvious asymptotic technique,

$$
f(x)=F(x)-\frac{v_{x}}{A n} \frac{\partial F}{\partial x}+\frac{v_{x}}{A n} \frac{\partial^{2} F}{\partial x^{2}}-\ldots
$$

This is, of course, just the Chapman-Enskog series, the second term corresponding to Navier-Stokes, the third to Burnett, etc. Iterating on the Navier-stokes solution as described earlier, we see that the departures noticed on the hot side could possibly be due to the higher order terms of $(7)$, especially the Burnett tyarms. The fact that the iteration scheme is essentially evaluating higher derivatives of $F$ is perhaps partiy responsible for the relatively slow convergence noticed on the hot side. The typical length scale of the profile $\delta_{2}$ on the hot side of the shock remains $\Lambda_{2}$ to a first approximation:

In a weak shock the Mach number $M=O(1)$ ejverywhere; and the argument made above for the hot side of a strong shock applies all through a weak shock.

The large deviations noticed on the cold side of strong shocks seem to be due to a different mechanism. Here $u=O\left(M_{1} \bar{c}_{1}\right)$. 
and the characteristic sampling distance $l_{1} \sim u_{1} / A_{1} n_{1} \sim M_{1} \Lambda_{1}$ becomes very large as $M_{1} \rightarrow \infty$, we see that $f$ is now determined as an integral over the flow and cannot possibly be described in terms of local derivatives (as in $(7)$ ). Also we may expect the characteristic length scale on the cold side to become $L_{1}$; for, if: we should put in for $F$ the Navier-stokes values (whose scale would be $\Lambda_{1} / \mathrm{M}_{1}$ ), the attenuation factor multiplying $F$ in ( 6 ) would immediately "smudge" it over a distance $\ell_{1} \cdot$. We conclude that the characteristic dimension of the 'tail.' is $\delta_{1} \sim M_{1} \Lambda_{1}$, and not $\Lambda_{1} / M_{1}$ as in the $N-s$ theory. A crude measure of the asymmetry of the profile is thus the ratio

$$
\frac{\delta_{1}}{\delta_{2}} \sim \frac{t_{1}}{L_{2}} \sim \mathrm{M}_{1} \frac{\Lambda_{1}}{\Lambda_{2}}
$$

If we take $\mu \sim T^{\omega}$, and define the mean free path as $\Lambda \sim \mu / \rho \bar{c}$ we have

$$
\frac{\delta_{1}}{\delta_{2}} \sim M_{1}\left(\frac{T_{1}}{T_{2}}\right)^{\omega-\frac{1}{2}} \sim M_{1} 2(1-\omega) .
$$

recalling that $\mathrm{T}_{2} / \mathrm{T}_{1} \sim \mathrm{M}_{1}^{2}$ for large $\mathrm{M}_{1}$. P It is easy to show (frum epe. 3 that the characteristic scales in the Navier-Stokes solution are proportional to the respective viscosities on either side of the shock; hence

$$
\left(\frac{\delta_{1}}{\delta_{2}}\right)_{N-S} \sim\left(\frac{T_{1}}{T_{2}}\right)^{\omega} \sim M_{1}^{-2 \omega}
$$


Since for real gases $/ / 2<\omega<1,\left(\delta_{1} / \delta_{2}\right)_{N-S} \rightarrow 0$, as $M_{1} \rightarrow \infty$ while for the $B-G-K$ model $\left(\delta_{1} / \delta_{2}\right) \rightarrow \infty$; Thus the Navier-stokes

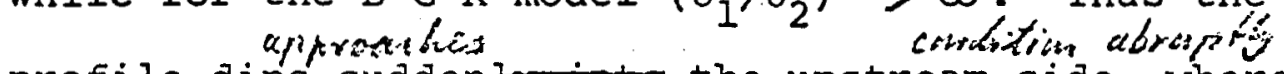
profile dipssiden into the upstream side, whoreas the B-G-K exact solution reaches the asymptotic state in a long, gradual tail as shown in Fig. He: It is further interesting to note that the $B-G-K$ tail becomes weaker as $\omega \rightarrow 1$, as noted in Figure 6 . This result for the ratio of the length scales $\dot{\delta}_{1} / \delta_{2}$ may be compared with the ratio of the appropriate free paths $\lambda\left(u_{1}\right) / \lambda\left(\bar{c}_{2}\right)$. For an intermolecular potential of the form $\varphi(r) \sim r^{-s}$, the free path $\lambda$ depends on the collision velocity $v$ obviously like $\lambda(v) \sim v^{4 / s}:$ Hence

$$
\frac{\lambda\left(u_{1}\right)}{\lambda\left(\bar{c}_{2}\right)} \sim \frac{u_{1}}{\overline{\bar{c}}_{2}} \sim M_{1}^{4 / n} \sim\left(\frac{T_{1}}{T_{2}}\right)^{\omega-\frac{3}{2}}
$$

and the ratio is thus, for large $\mathrm{M}_{1}$, nearly independent of $\mathrm{M}_{1}$. and only weakly dependent on $\omega$. The B-G-K model (except for the case of Maxwell molecules, i.e., $\omega=1$ ) is ${ }_{\lambda}$ likely to exaggerate the tail; the abrupt dip given by the $N-S$ solution on the other hand is certainly unrealistic and hence any solution of the Boltzmann equation' can be expected to fall between these two.

A great deal of interesting information is contained in the solution for $f$ of the model equation. When the profiles for the flow quantities within the shock have converged, they may be used to generate the distribution function, by evaluating the integral in (6). Many such computations have been carried out. and some typical results are shown in Figs. 5 and 6.

It is seen that over a fair part of the shock layer the distribution is strongly bimodal. The persistence of the "memory" 
of the supersonic stream is remarkable, and can be noticed even at $x=15$, where $\rho, u$ and $T$ have reached their downstream asymptotic values to one part in $10^{4}$ : This persistence is due to the longer free path of molecules traveling near the upstream velocity $u_{I}$, as discussed above; in fact the supersonic stream behaves like a molecular beam being attenuated by the molecules in the rest of velocity space. Qualitatively this behavior is not unexpected and has been used in models for the shock structure, Whitent busty 8ef. 10

e.g., by Rott and

There is a similar effect on molecules coming from the

subsonic stream, but this is much weaker on account of the smaller free paths.

The Chapman-Enskog distribution function even $M_{1}<2$ certainly differs locally considerably from the correct $f$ but does give the lower moments $h, u$ and $T$ with good accuracy.

TV. EXPERIMENTS

Only the density profiles of strong shocks have so:far been measured and the accuracy which has been reached is still of the order of $\pm 5 \%$ at best. Two methods have been used, Hornig's $(1 / f(2))$ method, in which the intensity of light refracted at nearly glancing incidence to the shock surface is measured, and the scattering of an electron beam which has been applied in various ways by a number of investigators. The most recent and complete measurements are the ones by Camac $(2 / j)$ and Russell ( $2 y / f)$.

Hornig.'s method depends on the phase differences in the light reflection from different regions of the shock profile and hence requires shock thicknesses of the order of a wave length of light. It has therefore the advantage of being applicable to thin shock 
waves and therefore to small shock tubes operating at high pressure. It has the serious drawback of being very sensitive to the angle of. . incidence and hence to the shock wave topography, which at high shock tube pressures can be quite complex (e.g., Ref./5). I.t is surprising indeed that the measurements are consistent within a few per cent and in good agreement with the other experiments.

The electron beam methods simply use the fact that for sufficiently low beam intensity scattered intensity is proportional to the number of scatterers. The intensity of the scattered beam is either measured directly like in Camac's' experiments using a high energy beam or obtained by measuring the "absorption" of a low energy beam like in the earlier measurements of Duff et.al..,and the recent ones of Russell. Due to the finite space and time resolution of the electron beam the method is best applied to thick shock waves and hence requires low pressure shock tubes (or wind tunnels) and relatively large ones to keep boundary layer induced curvature effects small.

A typical plot of the maximum density slope thickness versus $M_{l}$ is given in Fig. $T_{g}$. As a comparison computations based on the N-S approximation, B-G-K model and the Mott-Smith methods are shown. The agreement of the measurements with the $B-G-K$. model. computation is as good as can be expected. The agreement with the Mott-Smith computations is better, but then this is true for only one particular and arbitrary way of applying the method.

The most characteristic trend in the B-G-K profiles, the "tail" of the profile is very small for the density and probably anyway somewhat exaggerated by the model, consequently more detailed comparison of theory and experiment requires temperature profile or distribution function measurements. 
REFLRENCES

15) Bhatnagar, P. L., Gross, E. P. and Krook, M. (1954), Phys. Rev. 25, 521. (13) Camac, H. (2964), AIAA Aerospace Sciences Meeting, New York, Jenuary 20Preprint 64-35.

(j) Chahine, M. T. and Narasimbs, R. (1965), Proceedings of the 4th International Symposium on Rarefied Gas Dynamics, Toronto, Canade, 1964, J. E. de Leeuw, Baltor. (In Press)

(5) Gustafson, W. A. (1960), Phys. Fluids $3,732$.

(1) Ifepmann, H. W., Narasimba, R. and Chahine, M. T. (1952), Phys. Flutds 5 1313-1324.

(2) Liéubarski1, G. Ya (1961), Soviet Physics - JEIP 13740.

(12) Linzer, M., Bornig, D. F. (1963), Phys. Fluids 12, 1661-1668. and references yozen

(3) Mott-sinsth, घ. M. (1951), Phys. Rer. 82, 885-892. there.

(6) Nuckenfuss, c. (1962), Phys. Fluids $6,1325$.

(4) Rosen, P. (2954), J. Chem. Phys. 22, 1045.

(14) Russel2, D. (1965), Proceedings of the 4th International Symposium on Rarefied Cas Dynamics, Toronto, Cansda, 1054, J. H. de Leeur, Editor. (In Press)

(y) Solsurat, A. (1957), J. Flutd Mich. 3, 255-260.

(10)Rott N. and Hitlierfengy Darglan Rep. SH-38524 (1961) (II)Broadwell, J. E Phigh Flevide in press

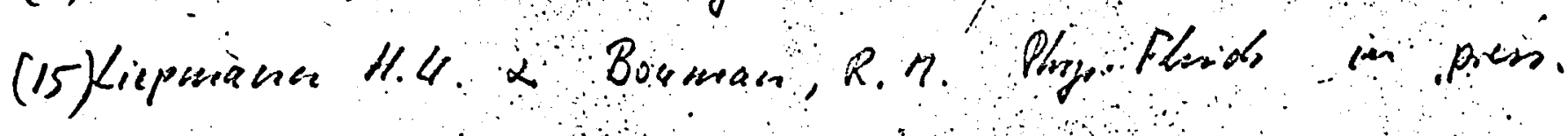




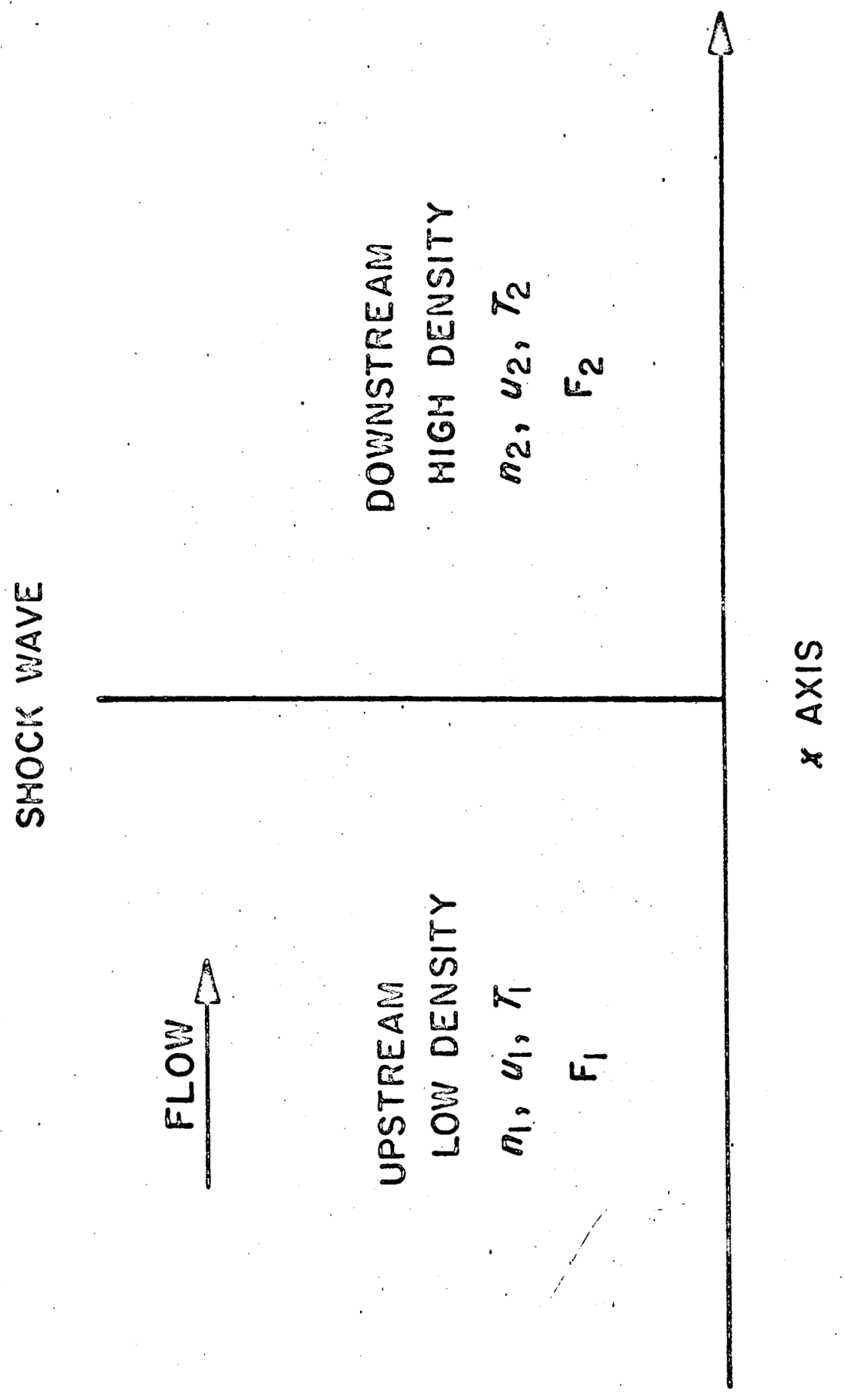




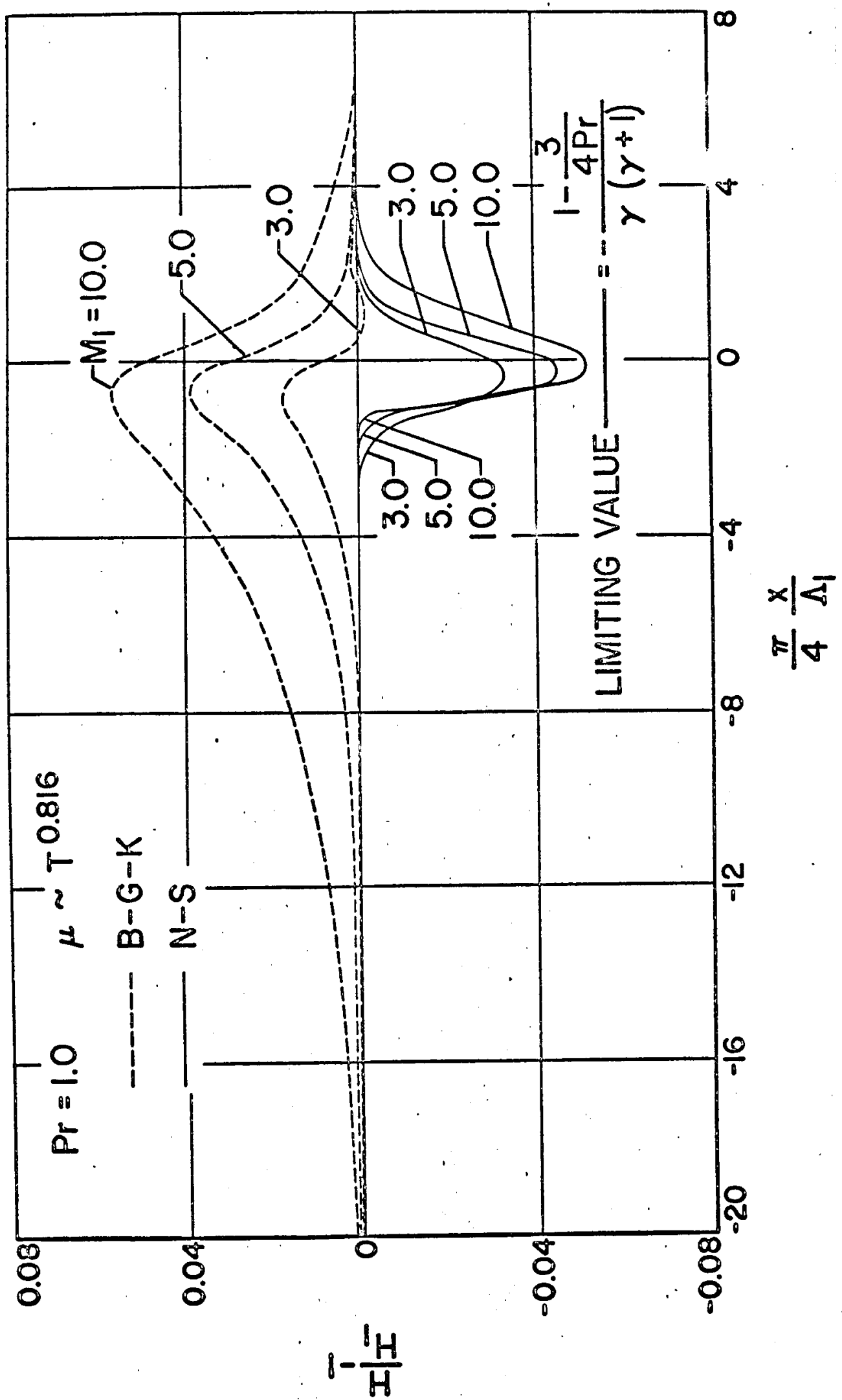




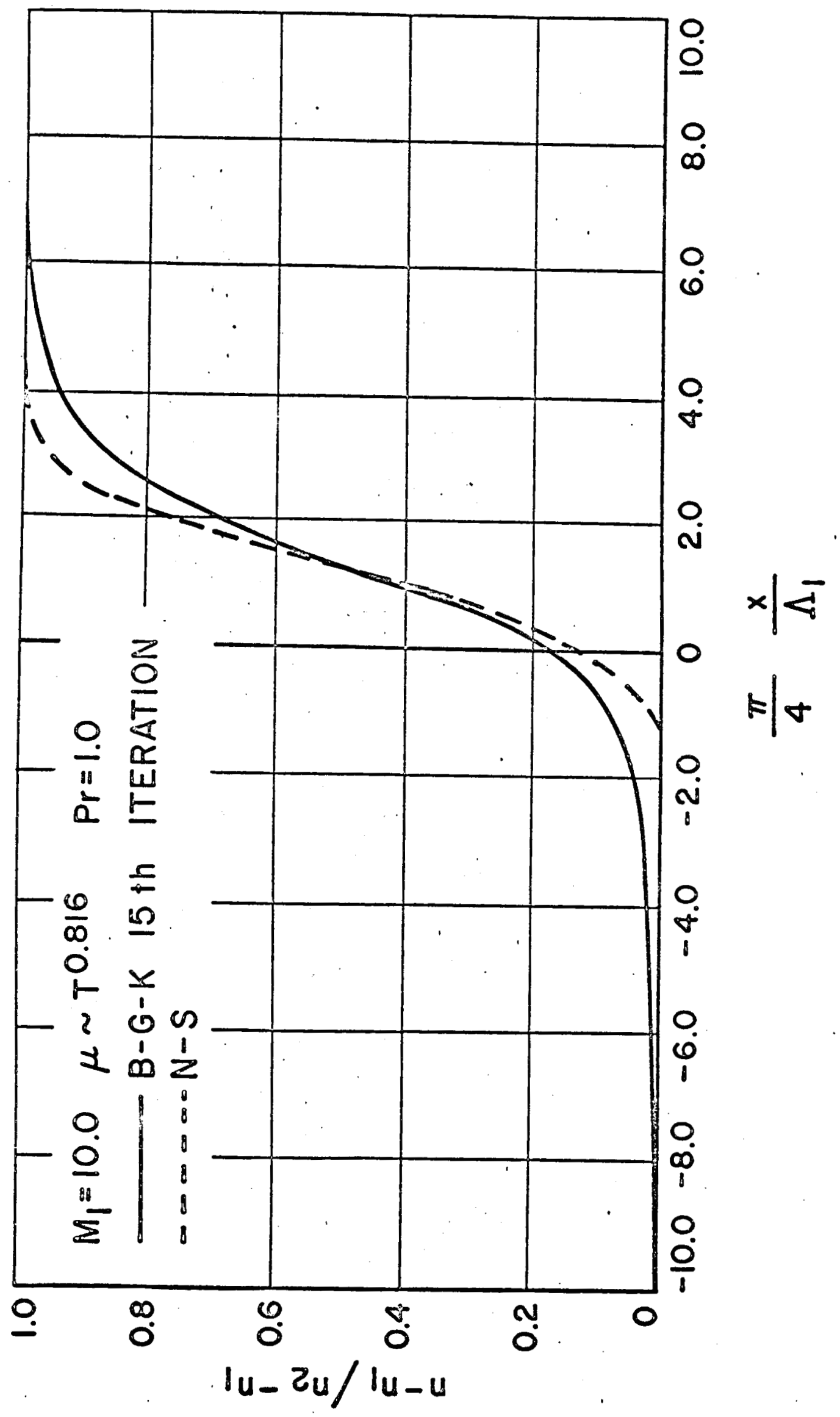




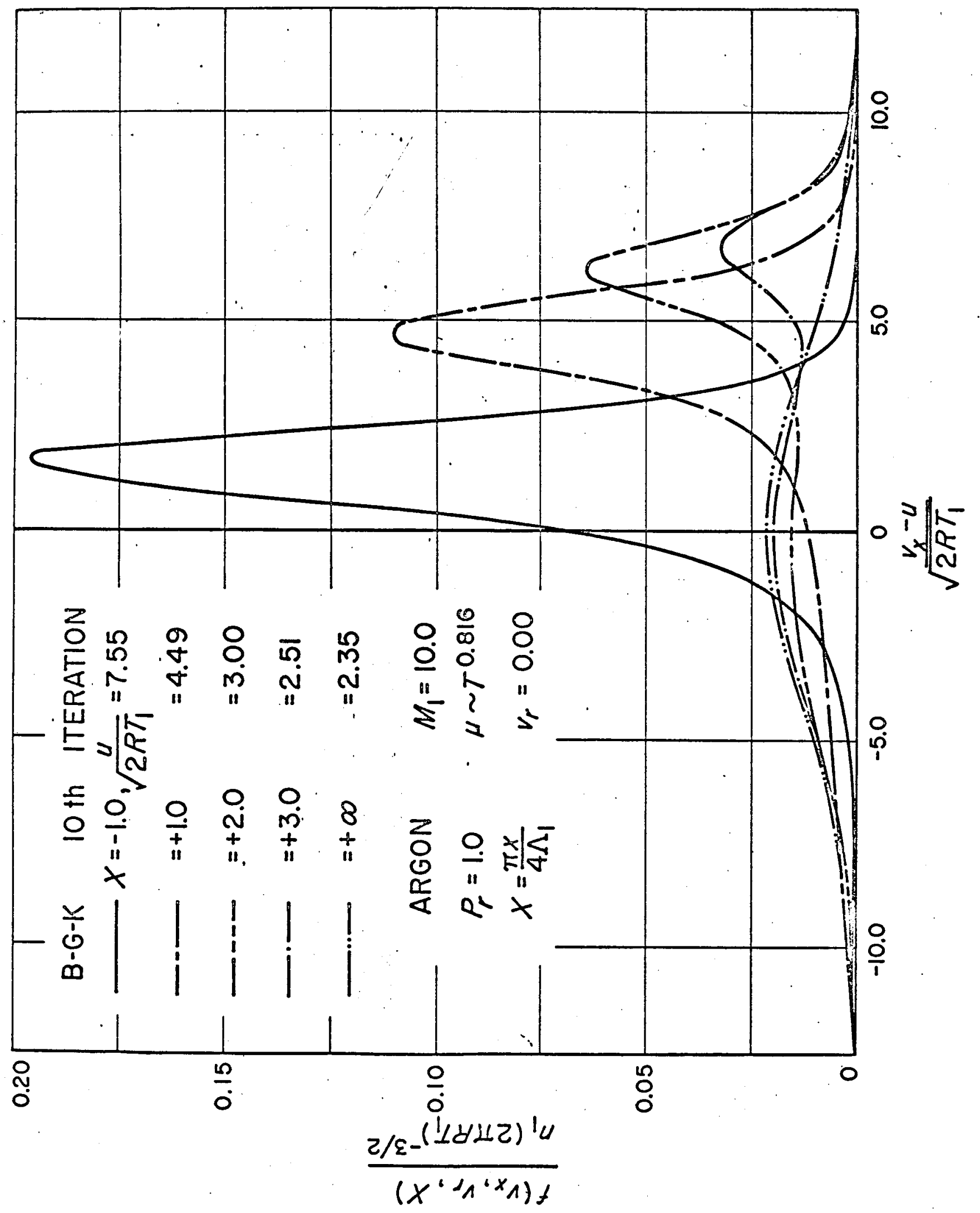




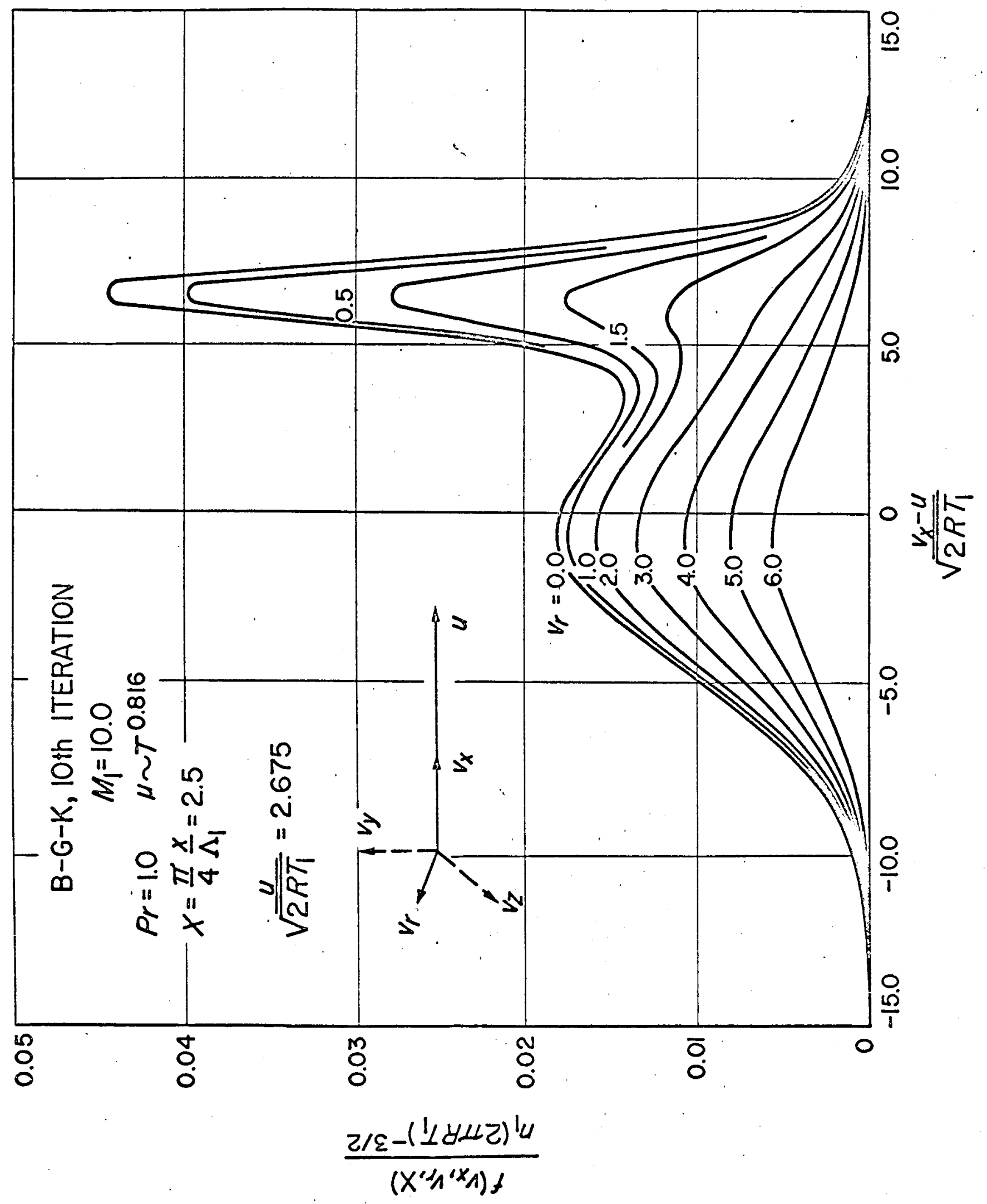




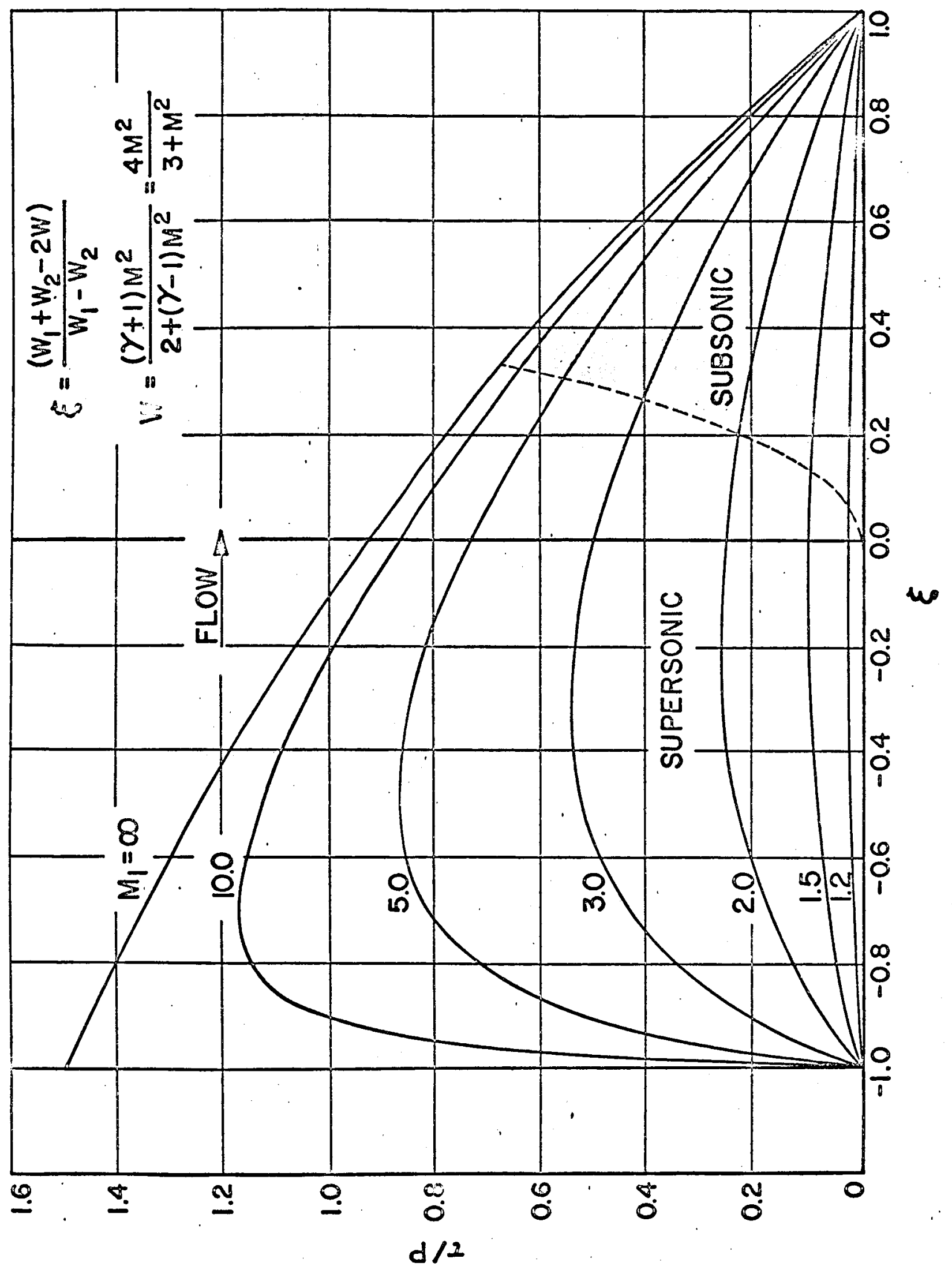

